\title{
THE TREATMENT OF GLYCOSURIA.
}

\author{
By T. H. OLIVER, M.D., F.R.C.P. \\ (Physician, Royal Infirmary, Manchester.)
}

Glycosuria is to be regarded as a symptom rather than a disease.

Before deciding, therefore, on the line of treatment to be adopted in a case where sugar has been discovered in the urine, not only must the possibility of a low renal threshold be excluded by a blood-sugar examination, but an attempt should be made to differentiate the conditions which may be primarily responsible for this symptom.

Every case of glycosuria is not pancreatic although there is frequently an associated disturbance of that organ. The primary lesion may lie in a disease of the central nervous system, encephalitis or cerebral tumour; even psychic disturbance may be responsible as there can be no doubt that anxiety or shock has a profound influence on the disease even where the primary lesion is elsewhere. Sometimes overaction of one of the other ductless glands is responsible. Acromegaly, hyperthyroidism or even adrenal overactivity may be the basic factor and the case cannot be adequately treated until its influence is taken into account. A severe anæmia should lead to a suspicion of pernicious anæmia, for the co-existence of the two diseases is not very uncommon, and the glycosuria may respond better with liver treatment than with insulin.

In extreme obesity, particularly in beer drinkers, the glycosuria appears to be the consequence of fatty changes in the liver and the glycosuria will disappear with a moderate restriction of diet and teetotalism. An enlarged hard liver suggests hæmochromatosis and the patient should be examined in a good light for pigmentation.

Indigestion is not common in diabetics and in those cases where flatulence is a prominent symptom, an associated cholecystitis should be suspected. Often the glycosuria will clear up completely after removal of the gall bladder. Diarrhœa in a diabetic is almost always due to an achlorhydria and both it and the glycosuria are often much improved after the administration of hydrochloric acid in full doses. In elderly patients a mild glycosuria which is associated with great loss of weight and strength is highly suspicious of malignancy, although the diagnosis cannot be made with certainty, until later in the disease. In the old, arteriosclerosis is one of the common accompaniments of diabetes mellitus and in many it appears to be the primary condition. The blood pressure, the condition of the arteries-particularly in the retinæ and in the feet-and the presence or absence of albuminuria should be observed carefully and the case may require treatment from this point of view rather than the diabetic. Again, gout and glycosuria are often associated and in my experience the diabetes is then of a mild type and responds better to dietetic than to insulin treatment. Lastly, sepsis, particularly in teeth and tonsils, should be sought for and tuberculosis be excluded if necessary by $\mathrm{X}$-ray examination. 
After this preliminary investigation, the first question to be decided is whether insulin should be used immediately or not. I regard the following as indications for its immediate use :-

(I) In children and young adults.

(2) The presence of acetone bodies in any type of case.

(3) Coma or threatened coma.

(4) Gangrene and carbuncle.

(5) Active tuberculosis.

(6) Diabetes mellitus associated with hyperthyroidism.

(7) Diabetes mellitus associated with severe nephritis.

(8) Diabetes mellitus associated with pregnancy.

In many cases after middle life it is impossible to determine immediately whether insulin will be required or not and I prefer to diet them for a few weeks before coming to a definite decision. If at the end of that time the patient is unable to take sufficient carbohydrate to maintain his strength without producing a glycosuria then insulin should be tried.

As diet is the basis of all treatment of diabetes mellitus, whether insulin is required or not, I will discuss it first and would emphasize the following principles :-

(I) The pancreas has a natural tendency toward recovery after rest, therefore a preliminary period of starvation is often helpful.

(2) The quantity of food taken is as important as the quality of the food, and it is useless to restrict carbohydrate if that restriction be compensated or overcompensated by an increase of protein and fat.

(3) The aim should be to keep the patient slightly undernourished. Thus where a normal man leading a sedentary life requires 2,600 to 3,000 calories, a diet of 2,300 to 2,400 calories should be aimed at for a diabetic. A woman requires about $4 / 5^{\text {ths }}$ of this amount.

(4) Excess of fat may lead to the development of arteriosclerosis as well as acetone bodies.

(5) A minimum of carbohydrate is necessary for health.

The necessity for carbohydrate is often unrealized. Sugar is essential for muscle function and if it cannot be obtained by the comparatively simple mechanism of the breakdown of starch, protein and probably fat will have to be the source of supply. This entails a complicated process which not only puts a great strain on metabolism but also leads to the production of toxic by-products.

In practice, I regard Ioo grms. of carbohydrate as the daily minimum. This is represented by 4 ozs. of white bread and 20 ozs. of $5 \%$ vegetables. If possible, I aim at giving considerably more than this and many patients can take 6 to $8 \mathrm{ozs}$. of ordinary bread a day without producing glycosuria. Often indeed a better tolerance results from this increase.

Patent breads are not usually required. They contain an undue proportion of protein and fat and are often unpalatable and expensive. 
Too much fat is a common error as patients are apt to think that it can be taken ad libitum and they do not realize that its high calorie value increases the total food intake enormously. It should not be forgotten that diabetics, like ordinary individuals, require less fat in hot weather and will require and metabolize an increase in the winter months. For the average case, I50 grms. daily is sufficient and this is contained in I I $\frac{1}{2}$ ozs. cooked bacon, I egg, 2 ozs. of cheese and 2 ozs. of cream and 2 ozs. of butter.

In the management of a case, I usually enjoin a preliminary period of starvation or semi-starvation for two to three days in order to reduce the blood sugar to normal and then put the patient on a diet of $\mathrm{I}, 000$ calories for a similar period. If the patient keeps sugar free, more food is gradually added until the limit of tolerance is reached.

I find it most convenient to estimate the carbohydrate tolerance as bread and when this has been found, other forms of carbohydrate can be substituted for it. Thus $\frac{1}{2}$ oz. may be replaced by 8 ozs. of $5 \%$ vegetables, $\frac{1}{2} \mathrm{oz}$. of rice or I $\frac{1}{2} \mathrm{ozs}$. of potatoes. $8 \mathrm{ozs}$. of milk is represented approximately by $\frac{1}{2} \mathrm{oz}$. of bread and $\frac{1}{2}$ oz. of butter.

Although such a scheme is not scientifically accurate, it is sufficiently so for practical purposes and does not lead to involved calculations. If the diet be too meticulous, the patient either tends to become an introspective neurotic or he breaks diet.

In the early stage of treatment it is best to keep the patient at rest and especially to try to shield him from worry. The nervous system has a profound influence on sugar metabolism and it is often the source of an unexpected relapse. Later moderate exercise should be encouraged and it will usually be found to have a very beneficial effect.

If insulin is decided upon, either because it is found to be impossible to give the minimum carbohydrate requirement without producing a recurrence of the glycosuria or because the case belongs to one of the categories mentioned above, it is desirable to form some estimate of what the result is likely to be.

This is often difficult and indeed it is impossible to say how much insulin will be required. It may be said, however, that children and young people usually react well and show a marked clinical improvement but stabilization may be difficult as the patient is likely to alternate rapidly between hypo- and hyperglycæmia.

Some of these cases react well to the slower acting protamine insulin and it is likely that the newer protamine zinc insulin will give even better results. Here, however, I would sound a note of warning. In the recent influenza epidemic, I have seen four cases of coma in patients treated with protamine insulin. Apparently it does not act quickly enough to withstand the effects of an infection and I now instruct them to take ordinary insulin again whenever they have a rise of temperature.

In adults, in my experience, the cases do best in which there is a history of a rapid increase of weight immediately prior to the wasting produced by the disease-especially those cases which show the typical brick-red complexion on a yellowish background. 
Cases associated with hyperthyroidism are difficult to stabilize until the thyroid has been removed, a proceeding which I always advocate if it be at all possible. In dealing with cases of hyperthyroidism it should be remembered that there is often a low renal threshold and in severe cases the blood-sugar curve may resemble that of a mild diabetic, so that it may not always be easy to decide whether the two diseases are present.

Tuberculosis used to be one of the most fatal of all the complications of diabetes mellitus, but since the introduction of insulin the results may be surprisingly good even in apparently hopeless cases. Here I find it best to give an ample diet without attempting to achieve stabilization. Even with considerable doses of insulin, 60 units a day, it is usually impossible to render the urine sugar free until the temperature has returned to normal. When the temperature has subsided a much smaller dose of insulin will probably be required and, if a reduction be not made, severe hypoglycæmic attacks may occur.

Since the introduction of insulin the combination of pregnancy and diabetes is a fairly common occurrence. Occasionally the disease first appears during pregnancy. The management of such cases may be difficult and periodic admission to hospital be necessary. As pregnancy tends to lower the renal threshold the urine is an unreliable guide, and the dose of insulin required can only be estimated by frequent blood sugar examinations. Usually an increased dose is necessary as pregnancy advances but occasionally the reverse is the case. In my opinion labour should always take place in hospital. Enquiry is often made as to the risks of pregnancy in a diabetic. Provided the patient can be supervised by a skilled obstetrician and the diabetes is properly controlled, the risk to the mother is very little above the normal. The risk to the child, however, is nearly? 50 per cent. The child is often unduly large, which makes labour difficult, and it is liable to suffer from congenital malformations. In many cases a Cæsarian section is advisable before term is reached. Inheritance of the disease by the child of a diabetic mother is uncommon and I have yet to meet an instance of it in my own practice.

The association of diabetes and nephritis is fairly common and often the renal condition is far the more important of the two. In such a case a reduction of the carbohydrate and the consequent proportional increase of protein and fat may so tax the damaged kidneys as to lead to disaster. Each case has to be judged on its merits but it is usually best to give ample carbohydrate, 8 or even Io ozs. of bread a day, which is compensated by a moderate dose of insulin. It may be inadvisable in many cases to attempt to get the urine sugar free.

Coma may occur in any type of diabetes. In young people it is the usual cause of death, but the liability to it gradually decreases with age and with the very old it is a rarity. Success in treatment depends on early recognition and the early signs cannot be emphasized too strongly:-

(a) Vomiting is almost invariable.

(b) Albumin as well as acetone appears in the urine.

(c) The breathing is altered and is deep and slow and the breath smells of acetone.

(d) Severe pain in the chest or abdomen may be an initial symptom.

(e) The tongue is dry and furred. 
Once the diagnosis has been made, treatment should be given immediately without waiting for a blood sugar estimation. 40 units should be given at once (in a severe case intravenously) and with it glucose, $\frac{1}{2}$ to I oz. in tea or lemonade. If at all possible the case should then be transferred to hospital, as the subsequent management of the case depends on repeated estimations of the blood sugar. The 40 units may have to be repeated in two hours' time but in most cases 20 units four-hourly is sufficient and glucose given by mouth if the patient can retain it. If vomiting is continuous, glucose salines or an intravenous injection of 5 per cent. glucose should be used. For the latter the continuous drip method is very successful. I do not think it matters how much glucose is given provided there is enough and no attempt should be made to render the urine sugar free at this stage.

Hypoglycæmia is a very real danger in the after-treatment of coma and as the renal threshold at these times may be unstable the urine is an unreliable guide. Special attention must be given to the bowels in these cases, a marked constipation is almost invariable which may require repeated enemata. A diminution in the amount of albumin is a hopeful sign, later the acetone decreases also and the tongue becomes moist. At this stage the glucose may be omitted and other forms of carbohydrate substituted and the insulin dropped to 20 units three times a day. The return to a full diet is gradually accomplished but it may be several weeks before stability is obtained.

In middle-aged and elderly people we meet a group which is difficult to manage and which is often associated with arteriosclerosis. Here the symptoms of diabetes are often slight and the patient comes under treatment for some other condition, gangrene, carbuncle or arteriosclerotic heart disease. These patients may fail to respond altogether to insulin therapy or the response may be only a partial one. In them, for reasons which I have discussed elsewhere ${ }^{(1)}$, I believe the lesion is not entirely pancreatic and the primary defect is an inability of the tissues to use the sugar.

Many cases can be controlled by dieting alone, others are helped by moderate doses of insulin, but a few either do not respond or are made worse, so that if large doses are given in an attempt to render them sugar free, they become definitely ill.

Although a fasting blood sugar level of $0.100 \%$ is the normal for a healthy person, it is not necessarily the optimum figure in disease and in practice it will be found that these patients often feel better when the blood sugar is considerably above the normal. Insulin should be used with caution therefore and reliance placed on the clinical response rather than the blood sugar figure. In my experience, this is particularly the case where there is arteriosclerotic heart disease and in these patients a low blood sugar, whether obtained by a strict noncarbohydrate diet or by insulin, produces a deterioration in the general condition and may be dangerous.

Often in these cases there is a raised renal threshold which I regard as a conservative process indicating that a low blood sugar figure is inadvisable.

In conclusion, I would emphasize that as the action of insulin is still unknown and the mechanism of the blood sugar level only partially understood, it is unwise to pin our faith entirely to laboratory results. Above all, the guiding principle should be to treat the patient rather than his urine, or even his blood sugar. 